2012

\title{
A Kinetic Model for Semidilute Bacterial Suspensions
}

\author{
Shawn D. Ryan \\ Cleveland State University, s.d.ryan@csuohio.edu \\ Leonid Berlyand \\ Pennsylvania State University \\ Brian M. Haines \\ Pennys/vania State University \\ D. A. Karpeev \\ Argonne National Laboratory
}

Follow this and additional works at: https://engagedscholarship.csuohio.edu/scimath_facpub

Part of the Mathematics Commons

How does access to this work benefit you? Let us know!

\section{Repository Citation}

Ryan, Shawn D.; Berlyand, Leonid; Haines, Brian M.; and Karpeev, D. A., "A Kinetic Model for Semidilute Bacterial Suspensions" (2012). Mathematics Faculty Publications. 307.

https://engagedscholarship.csuohio.edu/scimath_facpub/307

This Article is brought to you for free and open access by the Mathematics and Statistics Department at EngagedScholarship@CSU. It has been accepted for inclusion in Mathematics Faculty Publications by an authorized administrator of EngagedScholarship@CSU. For more information, please contact library.es@csuohio.edu. 


\title{
A KINETIC MODEL FOR SEMI-DILUTE BACTERIAL SUSPENSIONS *
}

\author{
S. D. RYAN ${ }^{\dagger}$, L. BERLYAND ${ }^{\dagger}$, B. M. HAINES ${ }^{\dagger}+$ AND D. A. KARPEEV
}

\begin{abstract}
Suspensions of self-propelled microscopic particles, such as swimming bacteria, exhibit collective motion leading to remarkable experimentally-observable macroscopic properties. Rigorous mathematical analysis of this emergent behavior can provide significant insight into the mechanisms behind these experimental observations; however, there are many theoretical questions remaining unanswered. In this paper, we study a coupled PDE/ODE system first introduced in the physics literature and used to investigate numerically the effective viscosity of a bacterial suspension. We then examine the kinetic theory associated to the coupled system, which is designed to capture the long-time behavior of a Stokesian suspension of point force dipoles (infinitesimal spheroids representing self-propelled particles) with Lennard-Jones-type repulsion. A planar shear background flow is imposed on the suspension through the novel use of Lees-Edwards quasiperiodic boundary conditions applied to a representative volume. We show the existence and uniqueness of solutions for all time to the equations of motion for particle configurations - dipole orientations and relative positions. This result follows from first establishing the regularity of the solution to the fluid equations. The existence and uniqueness result allows us to define the Liouville equation for the probability density of configurations. We show that this probability density defines the average bulk stress in the suspension underlying the definition of many macroscopic quantities of interest, in particular the effective viscosity. These effective properties are determined by microscopic interactions highlighting the multiscale nature of this work.
\end{abstract}

Key words. Collective Motion, Effective Viscosity, Coupled PDE/ODE System, Kinetic Theory

AMS subject classifications. 35Q35, 76D03, 76D07, 92B99.

1. Introduction. Pattern formation, anomalous rheology, and increased diffusivity are just a few of the many interesting macroscopic phenomena which have been observed in bacterial suspensions $[1,7,8,17,18,25-28,33]$. These properties result from the emergence of collective motion among the swimming bacteria and sharply distinguish suspensions of active particles from passive suspensions. Collective motion arises precisely because bacteria are self-locomoting microorganisms that move and interact through the fluid with each other and their environment.

One of the most striking experimental results in bacterial suspensions is the observation of a seven-fold reduction in the effective viscosity of a suspension of swimming Bacillus subtilis [26]. This reduction is seen as the volume fraction of bacteria increases to a critical value of about $2 \%$. Below this concentration threshold, referred to as the dilute regime, interactions between bacteria are negligible. It is sufficient to accurately describe the statistical state of the suspension by the probability of configurations - positions and orientations - of a single bacterium swimming in an externally-imposed background flow. This simplifying assumption has been used theoretically in $[10,12,21]$ and numerically in $[9]$ to explain the observed viscosity reduction. The reduction was shown to be the result of a coherent injection of momentum by the bacteria spending significant time aligned to the principal extensional axes of

${ }^{*}$ The work of S. D. Ryan, B. M. Haines, and L. Berlyand was supported by the Department of Energy grant DE-FG02-08ER25862.

${ }^{\dagger}$ Department of Mathematics, Pennsylvania State University, McAllister Bldg. University Park, Pennsylvania 16802, USA (ryan@math.psu.edu, berlyand@math.psu.edu)

${ }^{\ddagger}$ P.O. Box 1663, Mail Stop F644, Los Alamos National Laboratory, Los Alamos, NM 87545, USA (brianmhaines@gmail.com)

$\S$ Mathematics and Computer Science Division, Argonne National Laboratory, 9700 South Cass Avenue Argonne, IL 60439 USA (karpeev@mcs.anl.gov) 
the background flow. Here the equations governing the probability density of bacterial configurations can be explicitly derived and analyzed.

However, when the concentration exceeds $2 \%$, interactions between the bacteria become significant drivers of both interbacterial alignment and increased friction, contributing both to the decrease and increase in the effective viscosity. This was captured numerically through simulation in our prior work [20]. Analytically, the suspension of interacting particles is generally described by the probability density of configurations of all bacteria. The governing equations of the probability density are sometimes known as the kinetic theory of the suspension. Due to the presence of hydrodynamic interactions the kinetic theory will typically include singular terms, which diverge as bacteria approach each other. Further, when approximating a bulk suspension by a periodic system, only the relative motion (relative positions between each particle) of the suspended bacteria is meaningful. Up until now it has not been rigorously shown that such a kinetic theory is well defined and suitable to define an effective viscosity outside of the dilute limit.

In this paper we start to close this gap by analyzing a particular kinetic theory aimed at defining the effective viscosity for a semi-dilute bacterial suspension under a planar shear flow. Mathematically, we define a semi-dilute suspension as one that satisfies the superposition principle: the flow at any point in the fluid is the sum of the flows induced by the individual moving particles (see, e.g., [20]). This is also commonly known in the physics literature as mean field theory. We consider bacteria similar to $B$. subtilis used in the experimental work [26]. These bacteria are rod-like and propel themselves through the cumulative pushing action of their rotating helical flagella. The semi-dilute regime is defined by concentrations at which the interbacterial distances are typically much larger than the bacteria themselves, yet interactions between particles still play a prominent role. This allows the bacteria to be modeled as infinitesimal force dipoles acting on the fluid. This amounts to approximating the flow produced by each bacterium with its dipolar component [15], which captures the effect of its elongated shape and enforces the superposition principle. These assumptions are supported by experiment: recent work [8] shows that the flow from a swimming microorganism is well approximated by a dipolar flow at a distance, that the flow decays rather rapidly, and in the absence of significant alignment between the bacteria the flow is essentially negligible at distances comparable to the length of the bacterium. At shorter distances excluded volume interactions (collisions) dominate hydrodynamic interactions and are the main mechanism behind the initial alignment and the emergence of coherent motion. Therefore, we can expect that dipolar hydrodynamic interactions and Lennard-Jones-like excluded volume forces capture the main mechanisms of interbacterial interaction in the semi-dilute regime. In this paper we establish that the addition of excluded volume forces to the model serves to regularize the theory making it well defined and suitable for a definition of effective viscosity.

At a fixed time the instantaneous viscosity can be defined in terms of the volumeaveraged stress $[3,5]$, a function of only the instantaneous configuration of the suspended particles. The effective viscosity, on the other hand, must be a measurable macroscopic quantity independent of the particular particle configuration and, hence, of time. It can be defined using the long time average of the instantaneous viscosity, provided this limit exists and is independent of the initial configuration - the classical conditions for ergodicity - which can be challenging to establish in a rigorous mathematical framework. More basically, however, the kinetic theory must be well 
defined for all times in order for the long time limit to exist.

We define our kinetic theory as the Liouville equation for a suitable ODE governing the evolution of bacterial configurations. To ensure the Liouville equation is well defined for all time, the ODE's trajectories must exist for all time, for any initial condition in our phase space. The ODE is defined via a balance of forces and torques, which involves the hydrodynamic forces from the fluid governed by a PDE - the Stokes equation governing the flow generated by the force dipoles. We consider the specific yet representative case of a planar shear modeled by imposing time dependent quasiperiodic boundary conditions first proposed by Lees and Edwards [16]. With these boundary conditions and a given configuration of finitely many dipoles at a fixed time, the Stokes equation is explicitly solvable, at least in the weak sense due to the fact that the right-hand side is a distribution. A weak solution is insufficient due to the hydrodynamic forces entering the ODE, which require a pointwise evaluation of the fluid velocity and its derivatives. Additionally, because of the quasiperiodic form of the shearing boundary conditions, the resulting fluid velocity is not unique, but is defined only up to an additive constant. As a result, only the relative motion of the bacteria is well defined. To address these issues we establish the sufficient regularity of the PDE solution and show that the ODE, the kinetic theory as well as the viscosity can be self-consistently defined in terms of relative particle positions. Finally, we show that for any separated initial state - where particles have distinct initial positions - the ODE solution is defined for all time and remains separated. This allows us to conclude that the kinetic theory is also well defined and the investigation of its long time limit, including the question of effective viscosity, is meaningful.

The remainder of the paper is organized as follows. In Section $\S 2$ we discuss the definition of effective viscosity for bulk suspensions. Section $\S 3$ describes our model of the suspension as a coupled particle/fluid system as well as the modeling of shear. Section $\S 4$ establishes the main theoretical results: (i) the existence, uniqueness, and regularity of the solutions of the fluid equations, (ii) the global solvability of the particle equations of motion. Section $\S 5$ concludes the paper. Throughout this work, we adopt the Einstein summation convention and use the following notation for derivatives $u_{i, m}:=\frac{\partial u_{i}}{\partial x_{m}}$. To avoid confusion we denote vectors in the standard basis with a superscript, $\left\{\mathbf{e}^{1}, \mathbf{e}^{2}, \mathbf{e}^{3}\right\}$.

2. Effective (homogenized) viscosity of suspensions. Viscosity quantifies the response of the bulk fluid flow to an applied stress. Conversely, viscosity is a measure of the bulk stress in the fluid necessary to maintain a prescribed bulk flow. Assuming this response is linear, the viscosity is represented as a 4th order tensor, $\eta_{i j k l}$, quantifying the constitutive relationship between the deviatoric stress, $\Sigma_{i j}^{d}=$ $\Sigma_{i j}-\frac{1}{3} \delta_{i j} \Sigma_{m m}$, and the rate of strain, $E_{k l}$, as follows: $\Sigma_{i j}^{d}=\eta_{i j k l} E_{k l}$. In the case of an isotropic fluid the tensor is uniquely characterized by a single scalar, also called viscosity. In general, however, the relationship between the strain rate and the stress - the rheology of the fluid - is nonlinear and cannot be characterized by a constant scalar or tensor. Even in these situations, however, it is frequently possible to derive useful scalar constants quantifying some aspects of this constitutive relationship. Here we develop the definition of the effective shear viscosity as a measure of shear stress induced by a prescribed shear flow.

We impose a rate of strain on the suspension by specifying a planar shear background flow or, equivalently, suitable velocity boundary conditions, and compute the resulting bulk stresses acting in the suspension. A configuration of the suspension is defined as a set of bacterial positions $\underline{\underline{\mathbf{x}}}=\left(\mathbf{x}^{1}, \ldots, \mathbf{x}^{N}\right) \in \mathbb{R}^{3 N}$ and orientations 
$\underline{\mathbf{d}}=\left(\mathbf{d}^{1}, \ldots, \mathbf{d}^{N}\right) \in \mathcal{S}^{2 N}$, where $\mathcal{S}^{2}$ is the unit 2-sphere - the space of dipole orientations in $\mathbb{R}^{3}$. In contrast to the dilute regime where it was sufficient to consider two variables for a single bacterium's orientation, the added difficulty of a semidilute suspension is that now we must consider $5 N$ variables (three for each particle's position and two for each orientation). Assuming only dipolar and Lennard-Jones interactions as explained in Section $\S 1$, for a given configuration the instantaneous volume-averaged bulk stress is given by the symmetric trace-free tensor

$$
\Sigma_{l m}(\underline{\mathbf{x}}, \underline{\mathbf{d}})=\sum_{i=1}^{N}\left(\frac{U_{0}}{\left|V_{L}\right|}\left(d_{l}^{(i)} d_{m}^{(i)}-\frac{\delta_{l m}}{3}\right)+\sum_{j \neq i} \frac{r_{m}^{(i, j)} F_{l}\left(\mathbf{r}^{(i, j)}\right)}{\left|V_{L}\right|}\right)
$$

where $l, m=1,2,3, \mathbf{r}^{(i, j)}=\mathbf{x}^{i}-\mathbf{x}^{j}, U_{0}$ is the dipole moment, and $\left|V_{L}\right|$ is the volume of the domain. The stress is broken into two components: the hydrodynamic dipolar stress [3] and the excluded volume stress due to the Lennard-Jones force $\mathbf{F}$ [34]. The key feature of the stress is that it only depends on the $\frac{N(N-1)}{2}$ relative positions, $\mathbf{r}^{(i, j)}$ for $1 \leq i<j \leq N$, and $N$ orientations, $\mathbf{d}^{i}$. This is because in the dipole limit the total hydrodynamic stress is independent of the particles' positions, and the Lennard-Jones stress is defined in terms of relative positions. For a given background flow strength $\gamma$ and a given bacterial configuration at time $t$ we can calculate the instantaneous stress $\boldsymbol{\Sigma}(\underline{\mathbf{r}}, \underline{\mathbf{d}})$ and define the instantaneous shear viscosity as follows:

$$
\eta(\underline{\mathbf{r}}(t), \underline{\mathbf{d}}(t)):=\eta\left(1+\frac{\Sigma_{12}(\underline{\mathbf{r}}, \underline{\mathbf{d}})}{\gamma}\right),
$$

where $\eta$ is the velocity of the ambient fluid.

The viscosity is an intrinsic bulk property of a fluid. Bulk properties are characterized by the average behavior of the fluid on sufficiently large scales, such as those larger than the suspended particles or interparticle distances, while the local variations on smaller scales are irrelevant. Thus the effective viscosity, $\hat{\eta}$, like the viscosity of a homogeneous fluid should be a property of the suspension independent of time and the microscopic configuration. Thus we define the effective viscosity using a long time average of the instantaneous viscosity

$$
\hat{\eta}\left(\left(\underline{\mathbf{r}}_{0}, \underline{\mathbf{d}}_{0}\right)\right)=\eta\left(1+\lim _{T \rightarrow \infty} \frac{1}{T} \int_{0}^{T} \frac{\Sigma_{12}(\underline{\mathbf{r}}(t), \underline{\mathbf{d}}(t))}{\gamma} d t\right)
$$

similar to its computation in experiment. However, this quantity still depends on the initial configuration and in general is difficult to analyze. Instead, using the crucial assumption of ergodicity, we replace the time average by an ensemble average with respect to the steady-state probability distribution $P^{N}(\underline{\mathbf{r}}, \underline{\mathbf{d}})$ representing a statistically stationary state of the suspension

$$
\hat{\eta}=\eta\left(1+\int_{V_{L}} \int_{\mathcal{S}^{2}} \frac{\Sigma_{12}(\underline{\mathbf{r}}, \underline{\mathbf{d}})}{\gamma} P^{N}(\underline{\mathbf{r}}, \underline{\mathbf{d}}) d \underline{\mathbf{r}} d \underline{\mathbf{d}}\right) .
$$

Formally, the steady-state $P^{N}(\underline{\mathbf{r}}, \underline{\mathbf{d}})$ is the limit $t \rightarrow \infty$ of $P^{N}(\underline{\mathbf{r}}, \underline{\mathbf{d}}, t)$, which satisfies the Liouville equation

$$
\partial_{t} P^{N}=-\nabla_{\underline{\mathbf{r}}} \cdot\left(\underline{\dot{\mathbf{r}}} P^{N}\right)-\nabla_{\underline{\mathbf{d}}} \cdot\left(\underline{\dot{\mathbf{d}}} P^{N}\right)
$$




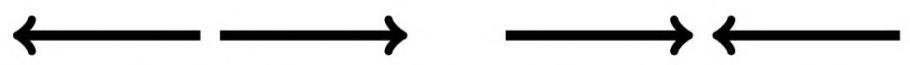

FIG. 3.1. Dipoles. Left: extensile dipole (pusher). Right: contractile dipole (puller).

where $\dot{\mathbf{r}}$ and $\dot{\mathbf{d}}$ are the relative equations of motion for the bacteria. To compute the effective viscosity it needs to be shown that the right-hand side of (2.2) is well defined as a function of the relative positions and orientations of the particles. The existence of a steady-state for (2.3) is not known in general and is the subject of future work. Here we take the first step in that direction by demonstrating that (2.3) is well defined for all time. To this end we need to analyze the ODE equations of motion of the bacteria and establish the long time existence of their solutions, which is the main focus of this work.

3. Model. Based on the prior discussion, we introduce the coupled PDE/ODE system modeling the bacterial suspension. The bacteria are represented as point force dipoles in a fluid. Heuristically, a dipole is the limit of infinitesimal separation of a pair of oppositely directed forces, which are equal in magnitude (see Fig. 3.1). One force represents the bacterium's propulsion force (the action of the flagella, for example), and the other is the opposing drag exerted on the fluid by the bacterium's body due to the no-slip boundary condition. This is a model of a "pusher", a swimmer that propels itself by pushing the fluid back behind its body as if with a propeller, similar to B. subtilis. "Pullers", which propel themselves with a kind of "breaststroke" by pulling the fluid in front of it (not considered here) can be modeled as contractile dipoles (Fig. 3.1 (right)). Based on the typical size $l_{0} \sim 1 \mu \mathrm{m}$ and swimming speed $V_{0} \sim 20 \mu \mathrm{m} / \mathrm{s}$ of a bacterium, as well as the typical dynamic viscosity $\sim 10^{-3} \mathrm{~Pa} \cdot \mathrm{s}$ and density $\sim 10^{3} \mathrm{~kg} / \mathrm{m}^{3}$ of the suspending fluid, the typical forces the bacteria exert generate flows with the Reynolds number, $R e \sim 10^{-4} \ll 1$. Similarly, inertial effects on the particle are negligible due to its small mass. We further assume that a steadystate flow is established on a timescale much smaller than that associated with the time for a bacterium to swim its length (characteristic timescale). Thus the fluid flow at any time is accurately modeled by the steady Stokes equation. It has been experimentally verified that the flow due to a force dipole in a Stokesian fluid is a good approximation to the flow around a swimming pusher [8], justifying our approximations.

To study effective viscosity we have to model the influence of a prescribed bulk background flow, as explained in Section $\S 2$. This problem is multiscale by nature: the effective properties such as the effective viscosity can only be defined on the macroscopic scale on the order of the size of the suspension, but are determined by the microscopic behavior on the scale of the particle size, $l_{0}$. On macroscopic length scales the flow in the suspension should be that of the background flow, but on the smaller length scales it will vary due the influence of the bacteria. Mathematically (see, e.g., [2]) the background flow is imposed on an unbounded system using suitable conditions at infinity, so that the result is free of finite boundary effects. This can be difficult to achieve numerically, however, as it would require very large computational domains. Instead, infinite bulk homogeneous systems are frequently modeled as periodic: the space is tiled with a lattice of periodic images of the fundamental cube $\square$, and the velocity and orientation of the fluid and particles in each cube are equal to those of their periodic images. If the size of the cube is much larger than the suspension's correlation distance, as in experiment [27], the statistics of the interparticle interactions will be similar to those in the bulk.

This approach, however, is not suitable for modeling shear flows, since the macro- 

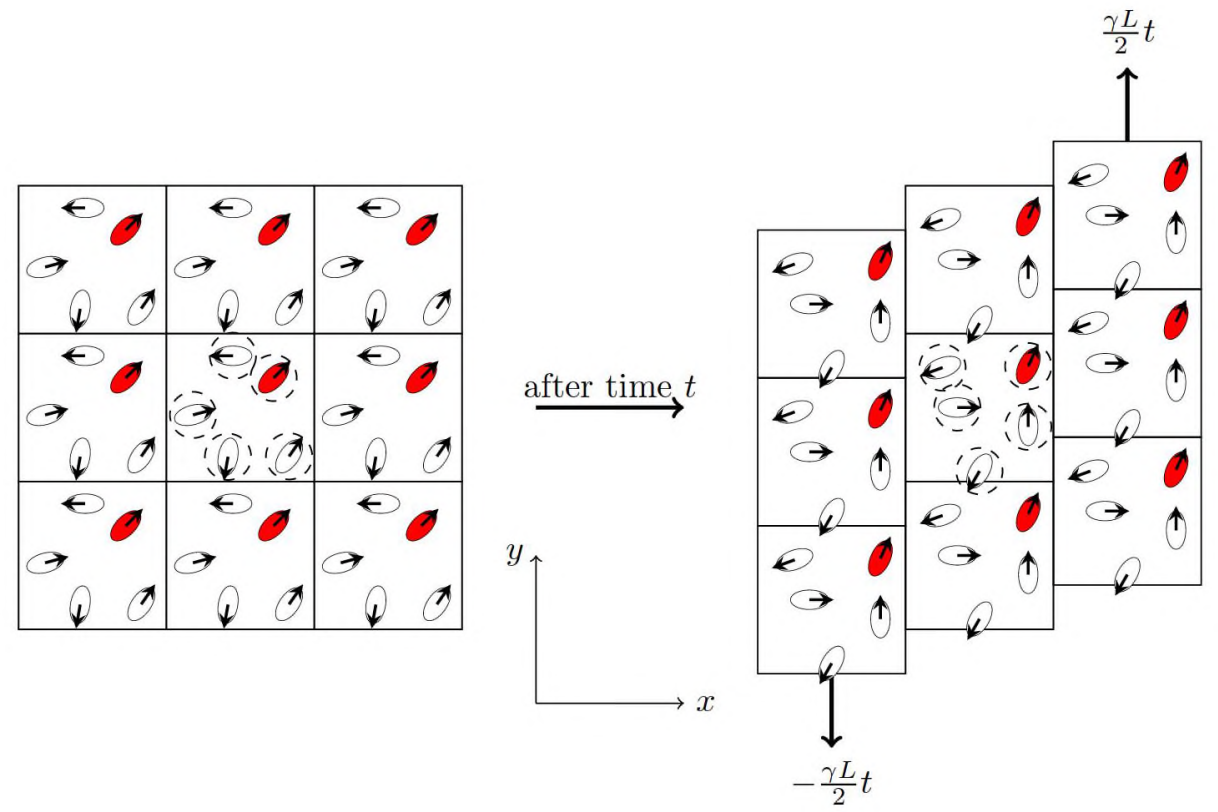

FIG. 3.2. Two-dimensional illustration of Lees-Edwards boundary conditions used to model planar shear flow.

scopic fluid velocity obtained by averaging over the cubes, will be constant. In order to ensure a linear variation in the velocity characteristic of shear we will employ an approach first proposed by Lees and Edwards [16] for molecular dynamics simulations of fluids. Assume for concreteness that the size of $\square$ is $L$, it is centered at the origin with the sides parallel to the coordinate axes. Initially there are $N$ periodically replicated particles in each cube. A shear of magnitude $\gamma$ is imposed in the $x y$-plane so that on large length scales the $y$-component of the flow varies as $\sim \gamma x$. We model this as follows: (a) at time $t$ each $y z$-layer of cubes is shifted upwards in $y$ by $\gamma t$ relative to the preceeding adjacent layer in $x$, and (b) in each subsequent $y z$-layer the $y$-component of the bacterial velocity is larger by $\gamma$. This way the global characteristics of the flow are those of planar shear, while the local interactions between the particles are as those in the bulk. In particular, the Lees-Edwards boundary conditions guarantee (see [6]) that the suspended particles in the image cells satisfy the same equations of motion as the particles in the reference cube $\square$, except with the velocity modified by the shear. This is not the case for periodic boundary conditions where the conservation of momentum may be violated [16]. For a two-dimensional illustration see Fig. 3.2.

We now develop a detailed mathematical description of this model. Observe that in the quasiperiodic setting set out above only the relative positions and velocities are meaningful. To simplify the presentation, however, we first derive the equations of motion for the absolute positions and orientations of the bacteria based on those of finite particles, and later convert them to the corresponding relative equations.

3.1. Equations of motion. The equations of motion for a force dipole naturally follow from those for an extended particle, which, given negligible inertial effects, are a consequence of the balance of forces and torques (see e.g., [11] for a derivation). 
Essentially, the force of self-propulsion is balanced by the hydrodynamic force, resulting in the Stokes drag law (3.1). In addition, particles interact with the flow as infinitesimal spheroids resulting in Jeffery's precessional equation (3.2) for an axisymmetric particle in a linear ambient flow. The semi-dilute assumption is increasingly more accurate as the size $l$ of the ellipsoidal bacterium shrinks, since the flow can be approximated by an increasingly more accurate linear Taylor expansion. The Stokes law determines the translation velocity $V_{0}$ of an isolated self-propelled spheroid for a given magnitude of the dipole moment $U_{0}=f_{0} \cdot l$ and eccentricity $e$ (see [11]; here $f_{0}$ is the magnitude of the self-propulsion force), and remains unchanged in the limit $l \rightarrow 0$ as long as $e$ and $U_{0}$ are kept fixed. The law given by Jeffery's equation, first investigated for the case of passive ellipsoids [13] and derived in [15], depends only on the eccentricity and remains unchanged in the limit. This dependence of rotational motion on the bacterial asymmetry is crucial to the alignment dynamies.

Each particle in a given cube, $\square$, moves in the ambient flow generated by the other particles. Because of the infinitesimal size of the dipoles, this ambient flow is the sum of flows $\mathbf{u}^{j}$ generated by each of the other $N-1$ dipoles in $\square$ as well as all of its quasiperiodic images. Additionally, we model excluded volume interactions or "soft collisions" by implementing Lennard-Jones-like forces $\mathbf{F}^{j}$ defined in Sec. 3.2, in contrast to pure hydrodynamic models $[22-24,29]$. A soft collision is a collision where kinetic energy is lost and takes into account excluded volume constraints in the sense that there is a radius of exclusion (see Sec. 4.3.1). These forces must balance self-propulsion and drag, so they enter into (3.1) but, being centrally-symmetric, contribute no torque and, therefore, are absent from (3.2). The Lennard-Jones terms have an artificial length scale characterizing the soft collision distance between the particles. Beyond this distance, chosen to be smaller than $L / 2$, the LJ forces vanish, so each particle interacts with no more than $N-1$ of the particles in the same $\square$ or its nearest images. Thus, we will interpret $\mathbf{F}^{j}\left(\mathbf{x}^{i}\right)$ as the force on the dipole $i$ in $\square$ from dipole $j$ in the same $\square$ or its closest quasiperiodic image. At most one of these particles exerts a nonzero force on $i$ (see Fig. 3.2), resulting in no ambiguity.

With this convention for the sums $\sum_{j \neq i}$, the resulting equations of motion are

$$
\begin{aligned}
& \dot{\mathbf{x}}^{i}=V_{0} \mathbf{d}^{i}+\sum_{j \neq i}\left(\mathbf{u}^{j}\left(\mathbf{x}^{i}\right)+\frac{1}{6 \pi \eta l_{0}} \mathbf{F}^{j}\left(\mathbf{x}^{i}\right)\right) \\
& \dot{\mathbf{d}}^{i}=-\mathbf{d}^{i} \times \sum_{j \neq i} \omega^{j}\left(\mathbf{x}^{i}\right)-\mathbf{d}^{i} \times\left[B \mathbf{d}^{i} \times \sum_{j \neq i} \mathbf{E}^{j}\left(\mathbf{x}^{i}\right) \cdot \mathbf{d}^{i}\right] .
\end{aligned}
$$

Here, $B$ is the Bretherton constant quantifying the aspect ratio of the particles. For a spheroid with eccentricity $e$ (the ratio of the major and minor axes) it is given by $B=\frac{e^{2}-1}{e^{2}+1},(B=0$ for spheres, $B=1$ for needles $)$, and for $B$. subtilis $B \approx .9$. The fluid velocity $\mathbf{u}^{j}$ generated by the $j$-th dipole is determined from the fluid equations (Sec. 3.3), and defines the corresponding vorticity $\boldsymbol{\omega}^{j}=\nabla \times \mathbf{u}^{j}$ and the strain-rate $\mathbf{E}^{j}=\frac{1}{2}\left(\nabla \mathbf{u}^{j}+\left(\nabla \mathbf{u}^{j}\right)^{T}\right)$. The dependence of these terms on the orientation $\mathbf{d}^{j}$ of the $j$-th particle is suppressed in the notation. The concentration, $\phi \sim N l_{0}^{3} /\left|V_{L}\right|$, is implicitly present in the coupled system due to the fact that the sums depend on the number of particles in the suspension, $N$, and the volume of the fluid domain $\left|V_{L}\right|$.

3.2. Lennard-Jones forces. As mentioned above, collisions between the bacteria are modeled as soft excluded volume interactions based on forces of the LennardJones (LJ) type. Specifically, $\mathbf{F}^{j}$ is the short-range repulsive force due to the $j$-th 


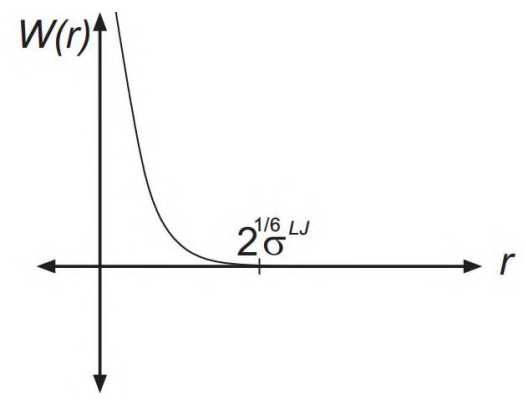

FIG. 3.3. Lennard-Jones type potential where $r=|\mathbf{x}|$ and $W(r)$ is radially symmetric.

particle and derived from the potential given by

$$
W(\mathbf{x})=\left\{\begin{array}{lr}
4 \varepsilon_{L J}\left[\left(\frac{\sigma_{L J}}{|\mathbf{x}|}\right)^{12}-\left(\frac{\sigma_{L J}}{|\mathbf{x}|}\right)^{6}\right]+\varepsilon_{L J}, & |\mathbf{x}| \leq 2^{1 / 6} \sigma_{L J} \\
0, & |\mathbf{x}|>2^{1 / 6} \sigma_{L J}
\end{array}\right.
$$

see Fig. 3.3. We then define $F^{j}\left(\mathbf{x}^{i}\right)=-\left.\nabla_{\mathbf{x}} W(\mathbf{x})\right|_{\mathbf{x}=\mathbf{x}^{2}-\mathbf{x}^{3}}$, where $\varepsilon_{L . J} \sim\left(\eta l_{0}^{2}\right)^{-1}$ is a constant determining the normalized strength of repulsion defined in terms of the real particle size $l_{0}$. The parameter $\sigma_{L J}$ determines the equilibrium distance $\left(2^{1 / 6} \sigma_{L, J}\right)$ where the net force between two bacteria balances to zero, and is set to reflect the real particle size: $\sigma_{L J} \sim l_{0}$. The truncation is imposed in order to ensure that this force is purely repulsive allowing soft collisions to be present. The truncated potential is only $C^{1}$-smooth (the constant shift $\varepsilon_{L, J}$, the minimum value of the Lennard-Jones 6-12 potential, is necessary to maintain continuity). One can still easily show that the LJ force $\mathbf{F}^{j}\left(\mathbf{x}^{i}\right)=\mathbf{F}\left(\mathbf{x}^{j}-\mathbf{x}^{i}\right) \in\left[C^{0,1}(\square \backslash\{0\})\right]^{3}$, where $C^{0,1}(\Omega)$ denotes the space of Lipschitz continuous functions on $\Omega$. This fact will be essential in Section $\S 4.3$ where the long time existence and uniqueness of particle trajectories is established.

3.3. PDE model for a force dipole in the fluid. To complete the definition of the equations of motion laid out in Sec. 3.1 we must define the flow $\mathbf{u}^{j}(\mathbf{x})$ generated at point $\mathbf{x}$ by the $j$-th dipole and its quasiperiodic images acting on the fluid. The total flow at any point is the sum of these individual dipolar flows in keeping with the semi-dilute assumption. Each dipole is subjected to an $x y$ planar shear flow of strength $\gamma$ used to define the effective viscosity (see Sec. 2). Shear flows are a representative linear flow frequently used in rheological experiments such as in [26]. We use the Lees-Edwards boundary conditions adapted to the PDE setting to model the effects of the shear. Specifically, the $j$-th dipole is oriented along $\mathbf{d}^{j}$ and located at $\mathbf{x}^{j}$ in a representative cube of side length $L$ centered at the origin $\square:=\{\mathbf{x} \in$ $\mathbb{R}^{3} \quad|\quad| x_{i} \mid<\frac{L}{2}$, for $\left.\mathrm{i}=1,2,3\right\}$. The fluid around the dipole satisfies the following Stokes equation:

$$
\left\{\begin{array}{lr}
\eta \triangle \mathbf{u}(\mathbf{x}, t)=\nabla p(\mathbf{x}, t)-\nabla \cdot\left[\mathbf{D}\left(\mathbf{d}^{j}\right) \delta\left(\mathbf{x}-\mathbf{x}^{j}\right)\right] & \mathbf{x} \in \square \\
\nabla \cdot \mathbf{u}(\mathbf{x}, t)=0 & \mathbf{x} \in \square \\
\mathbf{u}\left(-\frac{L}{2}, y-\frac{\gamma L t}{2}, z, t\right)=\mathbf{u}\left(\frac{L}{2}, y+\frac{\gamma L t}{2}, z, t\right)-(0, \gamma L, 0) & \mathbf{x} \in \partial \square \\
\mathbf{u}\left(x,-\frac{L}{2}, z, t\right)=\mathbf{u}\left(x, \frac{L}{2}, z, t\right), \quad \mathbf{u}\left(x, y,-\frac{L}{2}, t\right)=\mathbf{u}\left(x, y, \frac{L}{2}, t\right) & \mathbf{x} \in \partial \square
\end{array}\right.
$$


where $\mathbf{u}$ is the fluid velocity, $p$ is the pressure, and $\eta$ is the ambient fluid viscosity. The dipole tensor $\mathbf{D}$ is given by

$$
D_{j k}(\mathbf{d}):=U_{0}\left(d_{j} d_{k}-\frac{1}{3} \delta_{j k}\right)
$$

where $U_{0}<0$ is the dipole moment. To the best of our knowledge, the use of LeesEdwards boundary conditions in the formulation of a PDE or in the study of bacterial suspensions is novel.

3.4. Relative equations of motion. The flow governed by (3.4) is defined only up to a constant. Therefore, the right-hand side of the equations of motion (3.1)-(3.2) is not well defined. However, the main observable properties of the suspension, such as the stress (2.1) used to define viscosity, are only functions of the relative configuration of the particles. Therefore in place of (3.1)-(3.2) we reformulate the equations of motion for the $\frac{N(N-1)}{2}$ relative positions $\mathbf{r}^{(i, j)}:=\mathbf{x}^{i}-\mathbf{x}^{j}$ for $1 \leq i<j \leq N$ and $N$ orientations $\mathbf{d}^{i}$ for $1 \leq i \leq N$. This leads to $\frac{N(N-1)}{2}+N$ ordinary differential equations

$$
\begin{aligned}
& \dot{\mathbf{r}}^{(i, j)}=V_{0}\left(\mathbf{d}^{i}-\mathbf{d}^{j}\right)+\sum_{k \neq i}\left[\mathbf{u}\left(\mathbf{r}^{(i, k)}\right)+\mathbf{F}\left(\mathbf{r}^{(i, k)}\right)\right]-\sum_{k \neq j}\left[\mathbf{u}\left(\mathbf{r}^{(j, k)}\right)+\mathbf{F}\left(\mathbf{r}^{(j, k)}\right)\right] \\
& \dot{\mathbf{d}}^{i}=-\mathbf{d}^{i} \times\left(\sum_{j \neq i} \omega\left(\mathbf{r}^{(i, j)}\right)\right)-\mathbf{d}^{i} \times\left[B \mathbf{d}^{i} \times\left(\sum_{j \neq i} \mathbf{E}\left(\mathbf{r}^{(i, j)}\right)\right) \cdot \mathbf{d}^{i}\right]
\end{aligned}
$$

with initial conditions $\mathbf{r}^{(i, j)}(0)=\mathbf{x}_{0}^{i}-\mathbf{x}_{0}^{j}, \mathbf{d}^{i}(0)=\mathbf{d}_{0}^{i}$. Here $\mathbf{u}\left(\mathbf{r}^{(i, k)}\right)$ solves (3.4) with $\mathbf{d}=\mathbf{d}^{k}$.

The time evolution of the relative positions and orientation associated with $i$ th bacterium is completely determined by the relative positions and orientations of all other bacteria, and thus the system (3.6) - (3.7) is closed. As is clear from (3.4), $\mathbf{u}(\mathbf{x})$ is only defined up to an arbitrary constant, but this will cancel out in the reformulated equations. Recall from Section $\$ 2$ that these equations were necessary to show the Liouville equation for the probability density is well defined for all time. To calculate the effective viscosity, in addition to the probability distribution, we need the bulk stress to be well defined. To establish both results the long time existence of unique trajectories for the relative particle equations of motion (3.6)-(3.7) must now be shown.

4. Global Solvability of the equations of motion. We now turn to the central question of this paper - the existence of a unique solution to the equations of motion (3.6)-(3.7) for all time. There are two essential difficulties here. First, the right-hand side of (3.6)-(3.7) features $\mathbf{u}$ and its derivatives $\boldsymbol{\omega}, \mathbf{E}$. As a solution of the PDE (3.4) with a singular right-hand side and quasiperiodic boundary conditions, the velocity $\mathbf{u}$ might not be unique or have enough a priori regularity to have well defined pointwise values and derivatives. Second, under the best circumstances the regularity of $\mathbf{u}$ cannot extend to the collision set, $\mathbf{x}^{i}=\mathrm{x}^{j}$, where the flow due to the $j$-th dipoles is expected to diverge. Thus, in order to show long time existence, we have to establish the dynamics result that particle trajectories remain separated for all times, so that (3.6)-(3.7) remains Lipschitz.

We now examine the fluid velocity in greater detail. To establish the existence, uniqueness, and regularity of solutions to (3.4) we reduce it to the periodic case and 
later show how the general solution with Lees-Edwards boundary conditions can be obtained from the periodic case by a linear coordinate transformation.

4.1. Incompressible Stokes equation with periodic boundary conditions. We first define a few spaces which will be used throughout the remainder of this work. Denote the space of test functions, infinitely differentiable functions with compact support, as $\mathcal{D}\left(\mathbb{R}^{3}\right)$. We denote its dual space, the space of distributions, as $\mathcal{D}^{\prime}\left(\mathbb{R}^{3}\right)$. A dual pairing between a distribution $f \in \mathcal{D}^{\prime}\left(\mathbb{R}^{3}\right)$ and a test function $\phi \in \mathcal{D}(\mathbb{R})$ will be denoted by $(f, \phi)$. It is natural to consider solutions to (3.4) in the space of distributions due to the singularity on the right-hand side. We begin by considering the special case of a periodic flow. A periodic flow due a single dipole with orientation $\mathbf{d}$ at the origin satisfies the following equation in the distributional sense (i.e., as a pairing with arbitrary test functions).

$$
\begin{cases}\eta \Delta \mathbf{u}(\mathbf{x})=\nabla p(\mathbf{x})-\nabla \cdot[\mathbf{D}(\mathbf{d}) \delta(\mathbf{x})], & \mathbf{x} \in \square, \\ \nabla \cdot \mathbf{u}(\mathbf{x})=0, & \mathbf{x} \in \square, \\ \mathbf{u}(\mathbf{x}) \in\left[\mathcal{D}_{\square}^{\prime}\right]^{3} . & \end{cases}
$$

in $\mathrm{x} \in \square$. The space of periodic distributions on $\mathbb{R}^{3}$ is denoted by $\mathcal{D}_{\square}^{\prime}$ where the subscript $\square$ identifies the periodicity cell. Note here that divergence is to be understood in the distributional sense, $(\mathbf{u}, \nabla \phi)=0$ for all $\phi \in \mathcal{D}\left(\mathbb{R}^{3}\right)$. We begin by establishing the existence and regularity of the solutions to (4.1).

THEOREM 4.1. (Existence and Regularity of $\mathbf{u}(\mathbf{x})$ ) Given $\mathbf{d} \in \mathcal{S}^{2}$, there exists a solution $\mathbf{u}(\mathbf{x}) \in \mathcal{D}_{\square}^{\prime}$ to (4.1) and furthermore, $\mathbf{u}(\mathbf{x}) \in\left[C^{\infty}(\square \backslash\{0\})\right]^{3}$.

Proof. A periodic distribution is completely determined by the set of its Fourier coefficients [32]. Thus, we expand u. $p$, and $\mathbf{f}:=\nabla \cdot[\mathbf{D}(\mathbf{d}) \delta(\mathbf{x})]$ in Fourier series

$$
\mathbf{u}=\sum_{\mathbf{k} \in \mathbb{Z}^{3}} \mathbf{u}_{\mathbf{k}} e^{2 \pi i \mathbf{k} \cdot \mathbf{x}}, \quad p=\sum_{\mathbf{k} \in \mathbb{Z}^{3}} p_{\mathbf{k}} e^{2 \pi i \mathbf{k} \cdot \mathbf{x}}, \quad \mathbf{f}=\sum_{\mathbf{k} \in \mathbb{Z}^{3}} \mathbf{f}_{\mathbf{k}} e^{2 \pi i \mathbf{k} \cdot \mathbf{x}}
$$

analogous to [31] understood as distributions. Using the incompressibility condition $\mathbf{k} \cdot \mathbf{u}_{\mathbf{k}}=0$ one finds

$$
p_{\mathbf{k}}=\frac{\mathbf{k} \cdot \mathbf{f}_{\mathbf{k}}}{2 \pi i|\mathbf{k}|^{2}}, \quad \mathbf{u}_{\mathbf{k}}=-\frac{1}{4 \pi^{2}|\mathbf{k}|^{2}}\left[\mathbf{f}_{\mathbf{k}}-\frac{\left(\mathbf{k} \cdot \mathbf{f}_{\mathbf{k}}\right) \mathbf{k}}{|\mathbf{k}|^{2}}\right], \quad \mathbf{k} \in \mathbb{Z}^{3} \backslash\{\mathbf{0}\}
$$

Since $\mathrm{f}$ is a periodic distribution, its Fourier series converges to $\mathrm{f}$ in $\mathcal{S}^{\prime}\left(\mathbb{R}^{3}\right) \subset \mathcal{D}^{\prime}\left(\mathbb{R}^{3}\right)$, the space of tempered distributions on $\mathbb{R}^{3}$. In addition, for $\mathbf{k} \neq \mathbf{0},\left|p_{\mathbf{k}}\right| \leq\left|\mathbf{f}_{\mathbf{k}}\right|,\left|\mathbf{u}_{\mathbf{k}}\right| \leq$ $\left|\mathbf{f}_{\mathbf{k}}\right|$. Thus, the Fourier series (4.2) converge to $\mathbf{u}$ and $p$ in $\mathcal{S}^{\prime}$ and each is a periodic distribution (i.e., $(\mathbf{u}, p) \in\left(\left[\mathcal{D}_{\square}^{\prime}\right]^{3}, \mathcal{D}_{\square}^{\prime}\right)$ ). Thus a solution to (4.1) exists in the distributional sense.

Next we establish the regularity of the solution outside the origin. The intuitive idea is that the solution $(\mathbf{u}, p)$ will only have singularities at the same points the righthand side of (4.1) does. For this we appeal to a result for hypoelliptic equations [32]. Namely, a linear differential operator with constant coefficients $\mathcal{L}$ is hypoelliptic if and only if for any open set $\mathcal{O}$ every solution $u(x)$ in $\mathcal{D}^{\prime}(\mathcal{O})$ of the equation $\mathcal{L} u=f$, where $f \in C^{\infty}(\mathcal{O})$ belongs to $C^{\infty}(\mathcal{O})$.

Apply the divergence free condition to Stokes equation in (4.1) to find

$$
-\Delta p=\nabla \cdot \mathbf{f}
$$


in the distributional sense. Since all elliptic operators are hypoelliptic and $\nabla \cdot \mathrm{f} \in$ $C^{\infty}(\square \backslash\{0\})$, we find that $p \in C^{\infty}(\square \backslash\{0\})$. Now we consider Stokes equation for each component

$$
-\Delta u_{i}=-\frac{\partial}{\partial x_{i}} p+f_{i}, \quad i=1,2,3 .
$$

Applying the same result for Laplace's equation where the right-hand side belongs to $C^{\infty}(\square \backslash\{0\})$, we conclude that $\mathbf{u} \in\left[C^{\infty}(\square \backslash\{0\})\right]^{3}$ proving the regularity of solutions to (4.1) outside the origin.

An explicit construction of the solution $\mathbf{u}(\mathbf{x})$ to (4.1) can be found in Appendix A. Now that we have demonstrated the existence and regularity of a solution to equation (4.1), we prove that $(\mathbf{u}(\mathbf{x}), p)$ is the unique solution pair up to a constant.

Theorem 4.2. $(\mathbf{u}(\mathbf{x}), p(\mathbf{x})) \in\left(\left[\mathcal{D}_{\square}^{\prime}\right]^{3} \cdot \mathcal{D}_{\square}^{\prime}\right)$ is the unique solution to (4.1) up to a constant.

Proof. Assume there exists two solutions to (4.1), denoted $(\mathbf{u}(\mathbf{x}), p(\mathbf{x}))$ and $(\mathbf{v}(\mathbf{x}), q(\mathbf{x})) \operatorname{in}\left(\left[\mathcal{D}_{\square}^{\prime}\right]^{3}, \mathcal{D}_{\square}^{\prime}\right)$. Define $\mathbf{w}(\mathbf{x}):=\mathbf{u}(\mathbf{x})-\mathbf{v}(\mathbf{x})$ and the associated pressure $\phi(\mathbf{x}):=p(\mathbf{x})-q(\mathbf{x})$. Then $(\mathbf{w}(\mathbf{x}), \phi(\mathbf{x})) \in\left(\left[\mathcal{D}_{\square}^{\prime}\right]^{3}, \mathcal{D}_{\square}^{\prime}\right)$ solves

$$
\begin{cases}\eta \triangle \mathbf{w}(\mathbf{x})=\nabla \phi(\mathbf{x}) & \text { for } \mathbf{x} \in \square \\ \nabla \cdot \mathbf{w}(\mathbf{x})=0, & \text { for } \mathbf{x} \in \square .\end{cases}
$$

Expand both $\mathbf{w}(\mathbf{x})$ and $\phi(\mathbf{x})$ in a Fourier series analogous to the proof of Theorem 4.1 and [31]. Using equation (4.3) with $\mathbf{f}_{\mathbf{k}}=0$ and $\left\{\mathbf{u}_{\mathbf{k}}, p_{\mathbf{k}}\right\}$ replaced by $\left\{\mathbf{w}_{\mathbf{k}}, \phi_{\mathbf{k}}\right\}$ one immediately concludes that for $\mathbf{k} \neq 0, \phi_{\mathbf{k}}=0$ and $\mathbf{w}_{\mathbf{k}}=0$. Therefore, $\mathbf{w}(\mathbf{x})$ and $\phi(\mathbf{x})$ are constants. Since a periodic distribution is uniquely determined by its Fourier coefficients, $\mathbf{u}(\mathbf{x})=\mathbf{v}(\mathbf{x})$ and $p(\mathbf{x})=q(\mathbf{x})$ is the unique solution pair to (4.1) up to an additive constant.

4.2. Incompressible Stokes equation with Lees-Edwards conditions. In this section we will define a linear coordinate transformation, which will provide a relation between the solution to (3.4) and the solution to the periodic problem (4.1) for which we have already established an existence and uniqueness result. Recalling the purpose of these time-dependent quasiperiodic boundary conditions, which was to account for the shear in the $x y$-plane, we then introduce the map

$$
\mathbf{f}_{L E}^{t}(\hat{\mathbf{x}}): \mathbb{R}^{3} \rightarrow \mathbb{R}^{3}, \hat{\mathbf{x}}=(\hat{x}, \hat{y}, \hat{z}) \mapsto\left[\begin{array}{c}
\hat{x} \\
\hat{y}+\gamma t \hat{x} \\
\hat{z}
\end{array}\right]=: \mathbf{x}=(x, y, z),
$$

which transports a fluid particle located at $(\hat{x}, \hat{y}, \hat{z})$ to its location at time $t$ if it were only advected by the background flow. This change of coordinates map is invertible with inverse

$$
\left(\mathbf{f}_{L E}^{t}\right)^{-1}(\mathbf{x}): \mathbf{x} \mapsto\left[\begin{array}{c}
x \\
y-\gamma t x \\
z
\end{array}\right]=\hat{\mathbf{x}}
$$

where the Jacobian and inverse Jacobian of the coordinate transformation are

$$
J_{t}=\left[\begin{array}{ccc}
1 & 0 & 0 \\
\gamma t & 1 & 0 \\
0 & 0 & 1
\end{array}\right], \quad J_{t}^{-1}=\left[\begin{array}{ccc}
1 & 0 & 0 \\
-\gamma t & 1 & 0 \\
0 & 0 & 1
\end{array}\right]
$$


We are now able to precisely formulate and prove the desired result.

TheOREM 4.3. Given $t \in(0, \infty)$ and $\mathbf{d} \in \mathcal{S}^{2}$, the PDE (3.4) has a unique solution $\mathbf{u}(\mathbf{x}) \in\left[\mathcal{D}^{\prime}\left(\mathbb{R}^{3}\right)\right]^{3}$; moreover, $\mathbf{u}(\mathbf{x}) \in\left[C^{\infty}(\square \backslash\{0\})\right]^{3}$.

Proof. Let $\mathbf{u}(\mathbf{x}, t)$ be the velocity of the fluid which occupies position $\mathbf{x}$ at time $t$. Let $\mathbf{v}(\mathbf{x}, t)$ be the unique solution (up to a constant) to the periodic problem (4.1) at a given time $t$, which has already been shown to exist. By using the Lagrangian description, $\mathbf{v}(\hat{\mathbf{x}}, t)=\frac{d}{d t} \hat{\mathbf{X}}(t)$ such that at time $t, \hat{\mathbf{X}}(t)=\hat{\mathbf{x}}$. We now consider the relationship between $\mathbf{u}$ and $\mathbf{v}$. Define $\mathbf{u}$ in the following way

$$
\begin{aligned}
\mathbf{u}(\mathbf{x}, t):=\frac{d}{d t}\left[J_{t} \hat{\mathbf{X}}(t)\right] & =\left(\frac{d}{d t} J_{t}\right) \hat{\mathbf{X}}(t)+J_{t} \frac{d}{d t} \hat{\mathbf{X}}(t) \\
& =\left(\frac{d}{d t} J_{t}\right) \hat{\mathbf{x}}+J_{t} \mathbf{v}(\hat{\mathbf{x}}, t) \\
& =\left(\frac{d}{d t} J_{t}\right) J_{t}^{-1} \mathbf{x}+J_{t} \mathbf{v}\left(J_{t}^{-1} \mathbf{x}, t\right) \\
& =(0, \gamma x, 0)+J_{t} \mathbf{v}\left(J_{t}^{-1} \mathbf{x}, t\right) .
\end{aligned}
$$

Since $(0, \gamma x, 0) \in\left[C^{\infty}(\square)\right]^{3}$ and it was proven that for a fixed time $t, \mathbf{v}(\mathbf{x}) \in$ $\left[\mathcal{D}^{\prime}\left(\mathbb{R}^{3}\right)\right]^{3} \cap\left[C^{\infty}(\square \backslash\{0\})\right]^{3}$, then $\mathbf{u}(\mathbf{x}) \in\left[\mathcal{D}^{\prime}\left(\mathbb{R}^{3}\right)\right]^{3} \cap\left[C^{\infty}(\square \backslash\{0\})\right]^{3}$.

What remains to be shown is that $\mathbf{u}$ defined in this way satisfies (3.4).

$$
\begin{aligned}
& \Delta \mathbf{u}(\mathbf{x}, t)-\nabla p(\mathbf{x}, t)+\nabla \cdot[D(\mathbf{d}) \delta(\mathbf{x})]= \\
& \quad J^{t}\left\{\Delta \mathbf{v}\left(J_{t}^{-1} \mathbf{x}, t\right)-\nabla p\left(J_{t}^{-1} \mathbf{x}, t\right)+\nabla \cdot\left[D(\mathbf{d}) \delta\left(J_{t}^{-1}(\mathbf{x})\right)\right]\right\}=0 .
\end{aligned}
$$

since $\mathbf{v}$ satisfies (4.1). Therefore, $\mathbf{u}$ satisfies Stokes equation. Next, we verify the incompressibility condition

$$
\nabla \cdot \mathbf{u}(\mathbf{x}, t)=J_{t}\left[\nabla \cdot \mathbf{v}\left(J_{t}^{-1} \mathbf{x}, t\right)\right]=0 .
$$

Consider the boundary condition in the $x$-direction

$$
\begin{aligned}
\mathbf{u}\left(-\frac{1}{2}, y-\frac{\gamma t}{2}, z, t\right)+\left(0, \frac{\gamma}{2}, 0\right) & =J_{t} \mathbf{v}\left(-\frac{1}{2}, y, z, t\right) \\
& =J_{t} \mathbf{v}\left(\frac{1}{2}, y, z, t\right) \\
& =\mathbf{u}\left(\frac{1}{2}, y+\frac{\gamma t}{2}, z, t\right)-\left(0, \frac{\gamma}{2}, 0\right) .
\end{aligned}
$$

Similarly, $\mathbf{u}\left(x,-\frac{1}{2}, z, t\right)=\mathbf{u}\left(x, \frac{1}{2}, z, t\right)$ and $\mathbf{u}\left(x, y,-\frac{1}{2}, t\right)=\mathbf{u}\left(x, y, \frac{1}{2}, t\right)$. Thus, u satisfies (3.4).

Due to the 1-1 correspondence between solutions to the fluid equations with LeesEdwards boundary conditions (3.4) and solutions to the periodic problem (4.1) via the map (4.5), we have proven that the solution to (3.4) is unique up to a constant. We next establish the main result of the paper, namely the global solvability of the ODE equations of motion. As mentioned earlier, these equations contain the solution $\mathbf{u}$ to (3.4). The fact that the solution is only unique up to a constant does not effect the global solvability of (3.6)-(3.7).

4.3. Global solvability for the relative equations of motion. In this section we will show the long time existence of the solution to the relative equations of 
motion (3.6)-(3.7). The first step to proving long time existence is using the PicardLindelöf Theorem to show the short time existence of a unique solution.

THEOREM 4.4. Given a distinct set of initial relative positions $\mathbf{r}^{(i, j)}(0)=\mathbf{x}_{0}^{i}-\mathbf{x}_{0}^{j}$ for $1 \leq i<j \leq N$ and orientations $\mathbf{d}^{i}(0)=\mathbf{d}_{0}^{i}$ for $i=1, \ldots, N$, there exists a constant $T>0$ such that the relative equations of motion (3.6)-(3.7) have a unique solution for times $t \in[0, T)$.

Proof. In order to invoke the Picard-Lindelöf Theorem we must establish that the right-hand side of (3.6)-(3.7) is Lipschitz continuous. This is an immediate consequence of the regularity result proven in Section $\S 4.2$ in Theorem 4.3. Namely, for all $\varepsilon>0, \mathbf{u}(\mathbf{x}) \in\left[C^{\infty}\left(\square \backslash B_{\varepsilon}(0)\right)\right]^{3}$ and consequently $\omega(\mathbf{x}), \mathbf{E}(\mathbf{x}) \in\left[C^{\infty}\left(\square \backslash B_{\varepsilon}(0)\right)\right]^{3}$. Also, by the definition of the short-range potential (3.3), $\mathbf{F}(\mathbf{x}) \in\left[C^{0,1}\left(\square \backslash B_{\varepsilon}(0)\right)\right]^{3}$. Thus, as long as the particle trajectories remain separated, $\left|\mathbf{r}^{(i, j)}\right|=\left|\mathbf{x}^{i}-\mathbf{x}^{j}\right|>\varepsilon$, then the right-hand side of (3.6)-(3.7) is smooth. Therefore, outside the collision set, $\left\{\mathbf{x}^{i}=\mathbf{x}^{j}\right\},(3.6)-(3.7)$ has a Lipschitz continuous right-hand side and is bounded on $\square$. Thus by the Picard-Lindelöf Theorem [30], there exists a $T>0$ such that a unique solution to (3.6)-(3.7) exists for all $t \in[0, T)$.

4.3.1. Long-time existence - energy approach. To prove long time existence we need to show that the interval of time guaranteed by Theorem 4.4 can be extend to all time, (i.e., $T=\infty$ ). $T$ can only be finite if the solution to (3.6)(3.7) blows up in finite time. This occurs only if two particles collide, $x^{i}(t)=x^{j}(t)$ for $t=T$. However, heuristically, when $\left|\mathbf{x}^{i}-\mathbf{x}^{j}\right|<2^{1 / 6} \sigma_{L J}$, the particles begin to strongly repel one another due to the short-range potential $\left(\mathbf{F}\left(\mathbf{x}^{i}-\mathbf{x}^{j}\right) \sim 1 /\left|\mathbf{x}^{i}-\mathbf{x}^{j}\right|^{13}\right)$ and thus should remain apart for all time. If not the potential energy of the system will blow up. The following theorem will establish this intuitive idea rigorously and prove the main result of this work.

THEOREM 4.5. Given a distinct set of initial relative positions $\mathbf{r}^{(i, j)}(0)=\mathbf{x}_{0}^{i}-\mathbf{x}_{0}^{j}$ for $1 \leq i<j \leq N$ and orientations $\mathbf{d}^{i}(0)=\mathbf{d}_{0}^{i}$ for $i=1, \ldots, N$, there exists a unique solution to the relative equations of motion (3.6)-(3.7) for all time $t \in[0, \infty)$.

Proof. The main idea of the proof is to show that the particle trajectories avoid the collision set

$$
\mathbf{C}:=\left\{\mathbf{r}=\left(\mathbf{r}^{(1,2)}, \ldots, \mathbf{r}^{(N-1, N)}\right) \in \mathbb{R}^{3 N(N-1) / 2} \mid \mathbf{r}^{(i, j)}=0 \text { forsome } 1 \leq \mathrm{i}<\mathrm{j} \leq \mathrm{N}\right\} .
$$

Consider the unique maximal trajectory of all relative positions $\overrightarrow{\mathbf{r}}(t) \in \Gamma=\mathbb{R}^{3 N(N-1) / 2} \backslash$ $\mathrm{C}$ starting at $\overrightarrow{\mathbf{r}}_{0} \in \Gamma$, which exists by Theorem 4.4 for $t \in[0, T)$. We need to establish that $T=\infty$ to prove the result.

Begin by defining a function representing the potential energy at time $t$

$$
E(t):=\sum_{i=1}^{N} \sum_{j>i}^{N} W\left(\mathbf{r}^{(i, j)}(t)\right),
$$

where $E:[0, T) \rightarrow \mathbb{R}$ and $W(\mathbf{r})$ is the short-range potential (3.3) defined in Section $\S 3.2$. As mentioned in Section $\S 3.1, W\left(\mathbf{r}^{(i, j)}(t)\right)$ is unambiguously defined as the interaction between the $i$ th particle and the nearest image of the $j$ th particle at time $t$.

Assume, by contradiction, that $T<\infty . T$ can only be finite if the solution to (3.6)-(3.7) blows up as $t \rightarrow T$. This can only occur if as $t \rightarrow T$, a collision occurs between a pair of particles in the following sense: $\operatorname{dist}(\overrightarrow{\mathbf{r}}(\mathrm{t}), \mathbf{C}):=\min _{\mathrm{i}, \mathrm{j}}\left|\mathbf{r}^{(1, \mathrm{j})}(\mathrm{t})\right| \rightarrow 0$. 
As $\mathbf{r}^{(i, j)}(t) \rightarrow 0$ for any $1 \leq i<j \leq N$, then $W\left(\mathbf{r}^{(i, j)}(t)\right) \rightarrow \infty$ and thus $E(t) \rightarrow \infty$. In addition to $E(t)$, we compute its derivative

$$
\frac{d E}{d t}=\sum_{i=1}^{N} \sum_{j>i}^{N} \nabla W\left(\mathbf{r}^{(i, j)}(t)\right)+\dot{\mathbf{r}}^{(i, j)}
$$

using the equations of motion for relative positions (3.6). As $\operatorname{dist}(\overrightarrow{\mathbf{r}}(\mathrm{t}), \mathbf{C}) \rightarrow 0$, then $\frac{d E}{d t} \sim-\frac{1}{\left(\mathbf{r}^{(i, j)}\right)^{20}} \rightarrow-\infty\left(\right.$ since $\left.\dot{\mathbf{r}}^{(i, j)} \sim \mathbf{F}=-\nabla W\right)$. Recall that $\nabla W=-\mathbf{F}$ is Lipschitz continuous outside the origin.

Thus by the continuity of $\frac{d E}{d t}(t)$ for $t \in[0, T)$, there exists a $\delta>0$ such that if $\operatorname{dist}(\overrightarrow{\mathbf{r}}(\mathrm{t}), \mathbf{C})<\delta$ for any $t \in[0, T)$, then $\frac{d E}{d t}(t)<0$. Since a collision occurs as time $t \rightarrow T$, there exists a $T_{0} \in(0, T)$ such that for any $t>T_{0}$, $\operatorname{dist}(\overrightarrow{\mathbf{r}}(\mathrm{t}), \mathbf{C})<\delta$. So for any $t \in\left(T_{0}, T\right]$ we have

$$
E(t)=E(0)+\int_{0}^{t} \frac{d E}{d t}(\tau) d \tau<E(0)+\int_{0}^{T_{0}} \frac{d E}{d t}(\tau) d \tau=E\left(T_{0}\right)<\infty
$$

since $\overrightarrow{\mathbf{r}}\left(T_{0}\right) \notin \mathbf{C}$. This is a contradiction since $E(t) \rightarrow \infty$ as $t \rightarrow T$.

The key observation is that as $\operatorname{dist}(\overrightarrow{\mathbf{r}}(\mathrm{t}), \mathbf{C}) \rightarrow 0, E(t) \rightarrow \infty$, but $\frac{d E}{d t} \rightarrow-\infty$. This leads to the contradiction by appealing to the continuity of $E(t)$. Thus, $T=\infty$ and there exists a unique maximal trajectory defined for $t \in[0, \infty)$ given any initial relative configuration of positions $\overrightarrow{\mathbf{r}}_{0} \in \Gamma$. As a result the relative positions of particles remain bounded away from the collision set by $\delta>0$. Note that the righthand side of the equations of motion consists of elements of $C^{\infty}\left(\square \backslash B_{\delta}(0)\right)$. Since the arguments of each function remain in the compact set $\square \backslash B_{\delta}(0)$, then the right-hand side remains Lipschitz continuous for all time. Thus by extending Theorem 4.4 we have a unique solution to (3.6)-(3.7) for all time.

5. Conclusions. In this work the coupled PDE/ODE model for a semi-dilute suspension of self-propelled microscopic particles, first introduced in [20], was shown to possess a unique solution for all time given non-overlapping initial data. Using this result we define a kinetic theory, in the form of the Liouville equation, which governs the time evolution of the system in phase space. This work was motivated by considering the macroscopic phenomenon, specifically the change in the effective viscosity, observed in experiments on bacterial suspensions. Previous works studying the effective viscosity have yet to establish that this quantity is a well defined macroscopic quantity for a semi-dilute bacterial suspension. By proving the kinetic theory is well defined, we begin to justify the explicit formula for the effective viscosity derived in our prior work [20].

While we have shown that the Liouville equation is well defined, many important questions remain. One of the primary difficulties was the time dependent quasiperiodic boundary conditions on the fluid equation imposed by the planar shear background flow. It has yet to be investigated how other ambient background flows leading to different boundary conditions on the fluid equation effect the solvability of the coupled system. Also, in order to define the effective viscosity we illustrated the two components necessary. The bulk stress needs to be well defined for all time, which has been shown in this work, and there must exist a steady-state solution to the Liouville equation. Unlike the Fokker-Planck equation, where the existence of a steady-state probability distribution is known [19], we cannot appeal to such a result for the Liouville equation. Future work may establish this fact and allow the effective viscosity 
to be well defined as a macroscopic quantity. Nevertheless, the development of the kinetic theory in this work could lead to the study of other effective properties, in addition to the effective viscosity, experimentally observed in semi-dilute suspensions.

Appendix A. Construction of an explicit (series) solution to the problem (3.4).

We begin as in Section $\S 4.1$ by first constructing a solution to the periodic problem (4.1). Then as before we can obtain the solution to the problem (3.4) with LeesEdwards conditions by a linear coordinate transformation outlined in Section $\S 4.2$. We construct such a periodic flow to (4.1) from similar solutions in $\mathbb{R}^{3}$ by adapting the periodization technique as explained, for example, in [14] or [4]. To that end we take the distributional solution of

$$
\left\{\begin{array}{l}
\eta \Delta \hat{\mathbf{u}}(\mathbf{x})=\nabla \hat{p}(\mathbf{x})-\nabla \cdot[\mathbf{D}(\mathbf{d}) \delta(\mathbf{x})], \\
\nabla \cdot \hat{\mathbf{u}}(\mathbf{x})=0, \\
\hat{\mathbf{u}}(\mathbf{x}) \rightarrow 0, \text { as } \quad|\mathbf{x}| \rightarrow \infty
\end{array}\right.
$$

in $\mathbb{R}^{3}$

$$
\hat{u}_{i}(\mathbf{x})=\frac{1}{8 \pi \eta} \sum_{j=1}^{3} \sum_{k=1}^{3} D_{j k}(\mathbf{d}) \mathcal{G}_{i j, k}(\mathbf{x})
$$

and construct a formal sum of its translates

$$
u_{i}(\mathbf{x}):=\sum_{\mathbf{z} \in \mathbb{Z}^{3}} \hat{u}_{i}(\mathbf{x}+\mathbf{z}) .
$$

The intuitive idea here is that we expect $u_{i}$ defined in this way to be periodic. Here $D_{j k}(\mathbf{d})$ is the dipole tensor as in (3.5) and $\mathcal{G}(\mathbf{x})$ is the Oseen tensor (see [15]) with derivatives

$$
\mathcal{G}_{i j, k}(\mathbf{x}):=-\frac{1}{|\mathbf{x}|^{3}} \delta_{i j} x_{k}+\frac{1}{|\mathbf{x}|^{3}}\left(\delta_{i k} x_{j}+\delta_{j k} x_{i}\right)-\frac{3}{|\mathbf{x}|^{5}} x_{i} x_{j} x_{k} .
$$

The decay inherited from $\mathcal{G}_{i j, k}$ is too slow, $\hat{u}_{i}(\mathbf{x}+\mathbf{z}) \sim 1 /|\mathbf{z}|^{2}$, as the above series for $u_{i}$ does not converge. The essential idea, however, is sound: if a function decays sufficiently fast, the series of its translates will converge absolutely and uniformly to a periodic limit. To achieve the convergence of a series of translates, its terms can be modified by arbitrary elements of the kernel of the homogeneous Stokes operator (e.g., constants or suitable linear functions), namely

$$
\bar{u}_{i}(\mathbf{x}):=\hat{u}_{i}(\mathbf{x})+\sum_{\mathbf{z} \in \mathbb{Z}^{3} \backslash\{0\}}\left[\hat{u}_{i}(\mathbf{x}+\mathbf{z})-\hat{u}_{i}(\mathbf{z})-\hat{u}_{i, p}(\mathbf{z}) x_{p}\right] .
$$

which converges absolutely and uniformly due to each summand being $\sim O\left(\frac{1}{\mid \mathbf{z}^{4}}\right)$. Besides convergence, the sum must be periodic. To this end we can ensure periodicity by enforcing that the derivatives of the constructed solution $u_{i, k}(\mathbf{x})$ integrate to zero over periodic contours $C^{j}$, which connect $\mathbf{x}$ and $\mathbf{x}+\mathbf{e}^{j}$ avoiding any point in $\mathbb{Z}^{3}$ for $j=1,2,3$. Here $\mathbf{e}^{j}$ denotes the unit vector in the $j$ th direction. Thus we need to modify the sum by an additional linear term to ensure periodicity.

$$
u_{i}(\mathbf{x}):=\hat{u}_{i}(\mathbf{x})+\sum_{\mathbf{z} \in \mathbb{Z}^{3} \backslash\{0\}}\left[\hat{u}_{i}(\mathbf{x}+\mathbf{z})-\hat{u}_{i}(\mathbf{z})-\hat{u}_{i, p}(\mathbf{z}) x_{p}\right]-\Pi_{i j} x_{j} .
$$


where

(A.4) $\Pi_{i j}=\int_{C^{j}}\left(\hat{u}_{i, k}(\mathbf{x})+\sum_{\mathbf{z} \in \mathbb{Z}^{3} \backslash\{0\}}\left[\hat{u}_{i, k}(\mathbf{x}+\mathbf{z})-\hat{u}_{i, k}(\mathbf{z})\right]\right) d x_{k}=\bar{u}_{i}\left(\mathbf{x}+\mathbf{e}^{j}\right)-\bar{u}_{i}(\mathbf{x})$.

It will be shown that $\Pi_{i j}$ is a constant matrix independent of $\mathbf{x}$ and that $\Pi_{i i}=0$. Once, proven we conclude that $\Pi_{i j} x_{j}$ is in the kernel of the homogeneous Stokes operator. We now proceed to prove this construction of $\mathbf{u}$ indeed gives a solution to the periodic problem (4.1).

TheOREM A.1. Given $\mathbf{d} \in \mathcal{S}^{2}, \mathbf{u}(\mathbf{x})$ solves (4.1) and furthermore $\mathbf{u}(\mathbf{x}) \in\left[\mathcal{D}_{\square}^{\prime}\right]^{3} \cap$ $\left[C^{\infty}(\square \backslash\{0\})\right]^{3}$

Proof. We begin by showing that $\mathbf{u}(\mathbf{x})$ in (A.3) is a well defined periodic function for all $\mathbf{x} \notin \mathbb{Z}^{3}$. This requires further understanding of $\Pi_{i j}$, namely we prove that it is a constant matrix independent of $\mathbf{x}$.

It can be easily shown, (e.g., see Appendix B) that $\bar{u}_{i, m}(\mathbf{x})$ defined in (A.2) is $\mathbf{k}$-periodic for all $\mathbf{k} \in \mathbb{Z}^{3}$. Thus, in particular, $\bar{u}_{i, m}\left(\mathbf{x}+\mathbf{e}^{j}\right)-\bar{u}_{i, m}(\mathbf{x})=0$. Since the gradient of $\Pi_{i j}$ is equal to zero, we find that $\Pi_{i j}=C_{i j}$ a constant matrix independent of $\mathbf{x}$. Now that each component is understood, $\mathbf{u}(\mathbf{x})$ is a well defined function as a uniform limit of partial sums for $\mathbf{x} \notin \mathbb{Z}^{3}$.

We next establish the regularity of $\mathbf{u}(\mathbf{x})$ outside the origin. Since $\mathbf{u}(\mathbf{x})$ is the uniform limit of functions in $\left[C^{\infty}(\square \backslash\{0\})\right]^{3}$, then we conclude that $\mathbf{u}(\mathbf{x}) \in[C(\square \backslash\{0\})]^{3}$. Furthermore, by the uniform convergence of the partial sums of $k$ th derivatives, $\mathbf{u}(\mathbf{x}) \in\left[C^{k}(\square \backslash\{0\})\right]^{3}, \quad \forall k>0$ and the regularity result follows.

It remains to show that the derivatives, $u_{i, k}$, integrate to zero over all periodic contours.

$\int_{C^{j}} u_{i, k}(\mathbf{x}) d x_{k}=\int_{C^{j}} \hat{u}_{i, k}(\mathbf{x})+\sum_{\mathbf{z} \in \mathbb{Z}^{3}}\left[\hat{u}_{i, k}(\mathbf{x}+\mathbf{z})-\hat{u}_{i, k}(\mathbf{z})\right] d x_{k}-\int_{C^{j}} \Pi_{i k} d x_{k}=\Pi_{i j}-\Pi_{i j}=0$

Thus, $\mathbf{u}(\mathbf{x})$ is periodic with periodicity cell $\square$, the unit cube.

To summarize we have shown above that $\mathbf{u}(\mathbf{x})$ is pointwise defined in $\square$ outside the origin. The distributional solution to (A.1), $\mathbf{u} \in\left[L_{l o c}^{1}\left(\mathbb{R}^{3}\right)\right]^{3}$. Since $\mathbf{u}(\mathbf{x})$ is the uniform limit of $\left[L_{l o c}^{1}\left(\mathbb{R}^{3}\right)\right]^{3}$ functions over any compact set, then it is also in $\left[L_{l o c}^{1}\left(\mathbb{R}^{3}\right)\right]^{3}$ and defines a regular distribution $\left(\mathbf{u}(\mathbf{x}) \in\left[\mathcal{D}^{\prime}\left(\mathbb{R}^{3}\right)\right]^{3}\right)$. In addition, the periodicity of $\mathbf{u}(\mathbf{x})$ as an $\left[L_{l o c}^{1}\left(\mathbb{R}^{3}\right)\right]^{3}$ function implies its periodicity as a distribution. Therefore, we conclude that $\mathbf{u}(\mathbf{x}) \in\left[\mathcal{D}_{\square}^{\prime}\right]^{3} \cap\left[C^{\infty}(\square \backslash\{0\}]^{3}\right.$.

Now we must show that $\mathbf{u}(\mathbf{x})$ is divergence free in the distributional sense. Since $\hat{\mathbf{u}}(\mathbf{x})$ is a solution of the incompressible Stokes equation (A.1), it is divergence free, and thus

$$
u_{i, i}(\mathbf{x})=\hat{u}_{i, i}(\mathbf{x})+\sum_{\mathbf{z} \in \mathbb{Z}^{3} \backslash\{0\}}\left[\hat{u}_{i, i}(\mathbf{x}+\mathbf{z})-\hat{u}_{i, i}(\mathbf{z})\right]-\Pi_{i i}=-\Pi_{i i} .
$$

Therefore, it must be shown that $\Pi_{i i}=0$. The following lemma will provide the result.

Lemma A.2. For $\Pi_{i j}, \bar{u}(\mathbf{x})$, and $\square$ defined above the following relation holds

$$
\Pi_{i j}|\square|=\int_{\partial \square} \bar{u}_{i}(\mathbf{x}) \nu_{j} d^{2} x
$$

where $\nu$ is the unit normal to the surface. 
REMARK A.3. The proof is similar to the corresponding lemma in [4], where a solution is constructed to the periodic Laplace equation. This is a technical lemma proven in Appendix $C$.

Using this lemma, a short computation using the definition of $\bar{u}$ and $\hat{u}$ directly shows that in fact $\Pi_{i i}|\square|=\int_{\partial \square} \bar{u}_{i}(\mathbf{x}) \nu_{j} d^{2} x=0$. Since $|\square|>0$, we conclude that $\Pi_{i i}=0$ and therefore $\mathbf{u}(\mathbf{x})$ is divergence free. Since the partial sums each solve the incompressible Stokes equations and the sum converges in distribution, then $\mathbf{u}(\mathbf{x})$ also solves the incompressible Stokes equations in the distributional sense.

By the uniqueness result proven in Theorem 4.2, this is the unique solution to (4.1) up to a constant. We then apply the linear coordinate transformation (4.5) from Section $\S 4.2$ to find the unique solution to (3.4). This construction provides additional insight to the problem. Namely, we recover additional information that the solution found in Section $\S 4.1$ is not only a periodic distribution, but is also a regular distribution. In addition, this explicit construction could be used for further analysis of properties associated to solutions of (3.4) in future work.

Appendix B. Periodicity of $\overline{\mathbf{u}}_{i, k}(\mathbf{x})$.

Periodicity for a series of translates, $\bar{u}_{i, k}$, can be established as follows. If a smooth function decays at infinity as $\phi(\mathbf{x})=\mathcal{O}\left(\frac{1}{|\mathbf{x}|^{3}}\right)$, then

$$
\psi(\mathbf{x})=\sum_{\mathbf{z} \in \mathbb{Z}}[\phi(\mathbf{x}+\mathbf{z})-\phi(\mathbf{z})]
$$

converges absolutely and uniformly, since the terms in the series decay as $\mathcal{O}\left(\frac{1}{|\mathbf{x}|^{4}}\right)$. Then, for any $\mathbf{k} \in \mathbb{Z}^{3}$ we can rearrange the two series in $\psi(\mathbf{x}+\mathbf{k})-\psi(\mathbf{x})$ and resum over expanding balls $|\mathbf{z}|<K$ of radius $K$ :

$$
\psi(\mathbf{x}+\mathbf{k})-\psi(\mathbf{x})=\lim _{K \rightarrow \infty} \sum_{|\mathbf{z}|<K}[\phi(\mathbf{x}+\mathbf{k}+\mathbf{z})-\phi(\mathbf{x}+\mathbf{z})] .
$$

For sufficiently large $K$ most terms within the above sum cancel, except those in the thin "boundary" shell defined by the symmetric difference $A_{K}:=\{\mathbf{z} \in \mathbb{Z}:|\mathbf{z}-\mathbf{k}|<K\} \triangle$ $\{\mathbf{z} \in \mathbb{Z}:|\mathbf{z}|<K\}$. The sum of these, however, decays rapidly. Indeed, let $B_{K}:=\{\mathbf{z}:$ $\left.K-\frac{|\mathbf{k}|}{2}<\left|\mathbf{z}-\frac{\mathbf{k}}{2}\right|<K+\frac{|\mathbf{k}|}{2}\right\}$ be an annular region covering $A_{K}$ as illustrated in Fig. B, and thus the number of points in the sets satisfy $\left|A_{k}\right| \leq\left|B_{k}\right| \leq C K^{2}$, where $C>0$ is a constant. Thus, we have the following estimate

$$
|\psi(\mathbf{x}+\mathbf{k})-\psi(\mathbf{x})| \leq \lim _{K \rightarrow \infty} \sum_{\mathbf{z} \in B_{K}} \frac{C^{\prime}}{\left.|K-| \frac{\mathbf{k}}{2}\right|^{4}} \leq \lim _{K \rightarrow \infty} \frac{C^{\prime \prime}}{\left.|K-| \frac{\mathbf{k}}{2}\right|^{4}} K^{2} \rightarrow 0,
$$

where $C^{\prime}$ and $C^{\prime \prime}$ are some positive constants, so $\psi(\mathbf{x}+\mathbf{k})-\psi(\mathbf{x})=0$ and the periodicity of $\psi$ follows.

Appendix C. Proof of technical lemma used in Theorem A.1.

Lemma C.1. For $\Pi_{i j}, \bar{u}(\mathbf{x})$, and $\square$ defined in Section $\S A$ the following relation holds

$$
\Pi_{i j}|\square|=\int_{\partial \square} \bar{u}_{i}(\mathbf{x}) \nu_{j} d^{2} x
$$




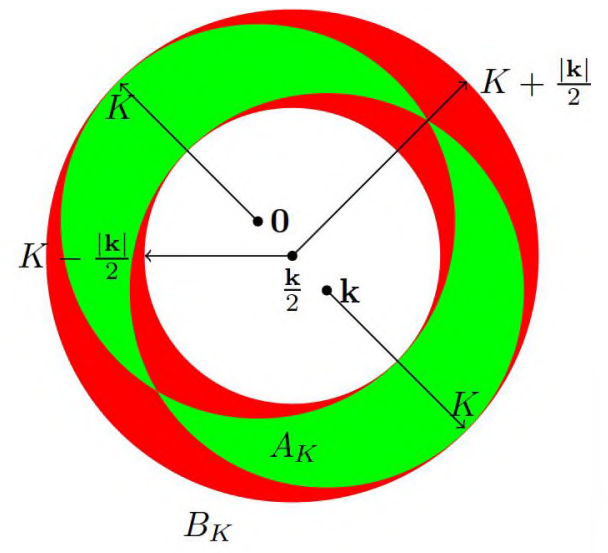

FIG. B.1. Schematic illustration of the multicolored annular region $B_{K}$ (red) covering the symmetric difference $A_{K}$ (green).

Proof. Let $\Gamma_{-}^{s}, \Gamma_{+}^{s}$ denote the opposing faces of $\square$ in the $s$ direction (normal to $\mathbf{e}_{s}$ ) and let $\nu$ denote the unit normal. First we establish the following equality:

$$
\int_{\partial \square} \bar{u}_{i}(\mathbf{x}) \nu_{j} d^{2} x=\sum_{s=1}^{3} \int_{\Gamma_{+}^{s}} \Pi_{i s} \nu_{j} d^{2} x .
$$

Let $\mathbf{y}=\mathbf{x}+\mathbf{e}_{s}$, under this change of variables $\Gamma_{-} \rightarrow \Gamma_{+}$and $\nu \rightarrow-\nu$. Thus,

$$
\int_{\Gamma_{+}^{s}} \bar{u}_{i}(x) \nu_{j} d^{2} x+\int_{\Gamma_{-}^{s}} \bar{u}_{i}(x) \nu_{j} d^{2} x=\int_{\Gamma_{+}^{s}} \bar{u}_{i}(x) \nu_{j} d^{2} x-\int_{\Gamma_{+}^{s}} \bar{u}_{i}\left(y-e_{s}\right) \nu_{j} d^{2} y=\int_{\Gamma_{+}^{s}} \Pi_{i s} \nu_{j} d^{2} x .
$$

By summing over all faces $s=1,2,3$ we establish (C.2).

Rewrite the right-hand side of (C.2),

$$
\text { (C.3) } \sum_{s=1}^{3} \int_{\Gamma_{+}^{s}} \Pi_{i s} \nu_{j} d^{2} x=\sum_{k, s, p=1}^{3}\left[\int_{\Gamma_{+}^{p}} \Pi_{i s} e_{k}^{s} x_{k} \nu_{j} d^{2} x-\int_{\Gamma_{+}^{p}} \Pi_{i s} e_{k}^{s}\left(x-e_{p}\right)_{k} \nu_{j} d^{2} x\right] \text {. }
$$

Using the above change of variables and the Divergence Theorem, (C.3) gives

$$
\sum_{s=1}^{3} \int_{\Gamma_{+}^{s}} \Pi_{i s} \nu_{j} d^{2} x=\sum_{k, s=1}^{3} \int_{\partial \square} \Pi_{i s} e_{k}^{s} x_{k} \nu_{j} d^{2} x=\int_{\square} \Pi_{i s} e_{j}^{s} d^{3} x=\Pi_{i j}|\square| .
$$

The result is proven.

\section{REFERENCES}

[1] I. S. Aranson, A. Sokolov, J. O. Kessler, and R. E. Goldstein, Physical Review E, 75 (2007), p. 040901.

[2] G. K. Batchelor, An Introduction to Fluid Dynamics, Cambridge University Press, 1967.

[3] G. K. Batchelor, J. Fluid Mech., 41 (1970), p. 545.

[4] V. Berdychevsky, Variational Principles Of Continuum Mechanics (in Russian), Nauka, 1983.

[5] H. BRENNER, Suspension rheology in the presence of rotary brownian motion and external couples: Elongational flow of dilute suspensions, Chem. Eng. Sci., 27 (1972), pp. 10691107. 
[6] K. DaYAl And R. D. James, Nonequilibrium molecular dynamics for bulk materials and nanostructures, J. Mechanics and Physics of Solids, 58 (2010), pp. 145-163.

[7] C. Dombrowski, L. Cisneros, S. Chatkaew, R.E. Goldstein, and J.O. Kessler, Physical Review Letters, 93 (2004), p. 098103.

[8] K. Drescher, J. Dunkel, L. Cisneros, S. Ganguly, and R. Goldstein, Fluid dynmamics and noise in bacterial cell-cell and cell-surface scattering, PNAS, 108 (2011), pp. 1094010945.

[9] V. Gyrya, K. Lipnikov, I. Aranson, and L. Berlyand, J. Math. Biol., (2010), pp. 1-34. 10.1007/s00285-010-0351-y.

[10] B. M. Haines, I. S. Aranson, L. Berlyand, and D. A. Karpeev, Phys. Biol., 5 (2008), p. 046003.

[11] - Effective viscosity of bacterial suspensions: A three-dimensional pde model with stochastic torque, CPAA, (2012).

[12] B. M. Haines, A. Sokolov, I. S. Aranson, L. Berlyand, and D. A. Karpeev, Physical Review E, 80 (2009), p. 041922.

[13] G. B. JEFFERY, The motion of ellipsoidal particles immersed in a viscous fluid, Proc. R. Soc. Lond. A, 102 (1922), pp. 161-179.

[14] V. V. Jikov, S. M. Kozlov, And O. A. OleINIK, Homogenization of Differential Operators and Integral Functionals, Springer-Verlag, 1994.

[15] S. Kim And S. J. Karilla, Microhydrodynamics: Principles and selected applications, Dover, 2005.

[16] A. W. Lees and S. F. Edwards, J. Phys. C, 5 (1972), p. 1921.

[17] K. C. Leptos, J. S. Guasto, J. P. Gollub, A. I. Pesci, and R. E. Goldstein, Physical Review Letters, 103 (2009), p. 198103.

[18] S. Rafaí, L. Jibuti, And Ph. Peyla, Physical Review Letters, 104 (2010), p. 098102.

[19] H. RISKEN, The Fokker-Planck equation: methods of solution and applications, Springer-Verlag, New York, 1989.

[20] S. D. Ryan, B. M. Haines, L. Berlyand, F. Ziebert, and I. S. Aranson, Viscosity of bacterial suspensions: Hydrodynamic interactions and self-induced noise, Physical Review E, 83 (2011).

(21] D. Saintillan, Extensional rheology of active suspensions, Physical Review E, 81 (2010), p. 056307.

[22] D. Saintillan AND M. J. SHelley, Orientational order and instabilities in suspensions of self-locomoting rods, Physical Review Letters, 99 (2007), p. 058102.

[23] _ Instabilities and pattern formation in active particle suspensions: Kinetic theory and continuum simulations, Physical Review Letters, 100 (2008), p. 178103.

[24] - Emergence of coherent structures and large-scale flows in motile suspensions, J. Royal Society Interface, (2011).

[25] A. Sokolov, M. M. Apodaca, B. A. Grzybowski, and I. S. Aranson, PNAS, 107 (2010), p. 969.

[26] A. Sokolov and I. S. ARanson, Reduction of viscosity in suspension of swimming bacteria, Physical Review Letters, 103 (2009), p. 148101.

[27] A. Sokolov; I. S. Aranson, J. O. Kessler, and R. E. Goldstein, Physical Review Letters, 98 (2007), p. 158102.

[28] A. Sokolov, R. E. Goldstein, F. I. Feldchtein, and I. S. Aranson, Enhanced mixing and spatial instability in concentrated bacterial suspensions, Phys. Rev. E, 80 (2009).

[29] G. Subramanian AND D. L. Koch, Critical bacterial concentration for the onset of collective swimming, J. Fluid Mech, 632 (2009).

[30] M. E. TAYlor, Partial Differential Equations: Basic Theory, Springer, 1996.

[31] R. TEMam, Navier-Stokes Equations and Nonlinear Functional Analysis, SIAM, 1995, ch. 2.

[32] V. S. Vladimirov, Methods of the Theory of Generalized Functions, CRC Press, 2002.

[33] X.-L. Wu And A. Libchaber, Physical Review Letters, 84 (2000), p. 3017.

[34] F. Ziebert and I. S. Aranson, Physical Review E, 77 (2008), p. 011918. 